\title{
INVERSE DESIGN OF MATERIALS THAT EXHIBIT THE MAGNETOCALORIC EFFECT BY TEXT-MINING OF THE SCIENTIFIC LITERATURE AND GENERATIVE DEEP LEARNING
}

\section{Supporting Information}

\author{
Callum J. Court ${ }^{1}$, Apoorv Jain ${ }^{1,2}$, and Jacqueline M. Cole ${ }^{1,2,3 *}$ \\ ${ }^{1}$ Cavendish Laboratory, Department of Physics, University of Cambridge, J. J. Thomson Avenue, Cambridge, CB3 \\ OHE, UK \\ ${ }^{2}$ Department of Chemical Engineering and Biotechnology, University of Cambridge, West Cambridge Site, Philippa \\ Fawcett Drive, Cambridge, CB3 OFS, UK \\ ${ }^{3}$ ISIS Neutron and Muon Source, STFC Rutherford Appleton Laboratory, Harwell Science and Innovation Campus, \\ Didcot, OXI1 0QX, UK \\ *Corresponding Author: jmc61@cam.ac.uk
}

August 29, 2021

\section{ChemDataExtractor 2.0 models}

Here we provide the source code for the creation of the CurieTemperature, MagneticEntropy, RelativeCoolingPower and MagneticField models. 
from chemdataextractor.model import BaseModel, ListType, ModelType, StringType from chemdataextractor.model.units.temperature import TemperatureModel

from chemdataextractor.model.units.energy import Energy

from chemdataextractor.model.units.mass import Mass

from chemdataextractor.parse.actions import join

6 from chemdataextractor.parse.auto import AutoTableParser

7 from chemdataextractor.parse.common import dt, lbrct, rbrct

8 from chemdataextractor.parse.elements import I, Optional, R, W, Zero0rMore

9 from chemdataextractor.parse.template import (MultiQuantityModelTemplateParser, QuantityModelTemplateParser)

11 from .inorganic_compound import InorganicCompound, inorganic_names_only

12 from .magnetic_field import MagneticField

delim $=R(" \wedge[: ; \backslash .] \$ "$,

\# Curie Temperature Specifier

curie_specifier $=($

$$
(
$$




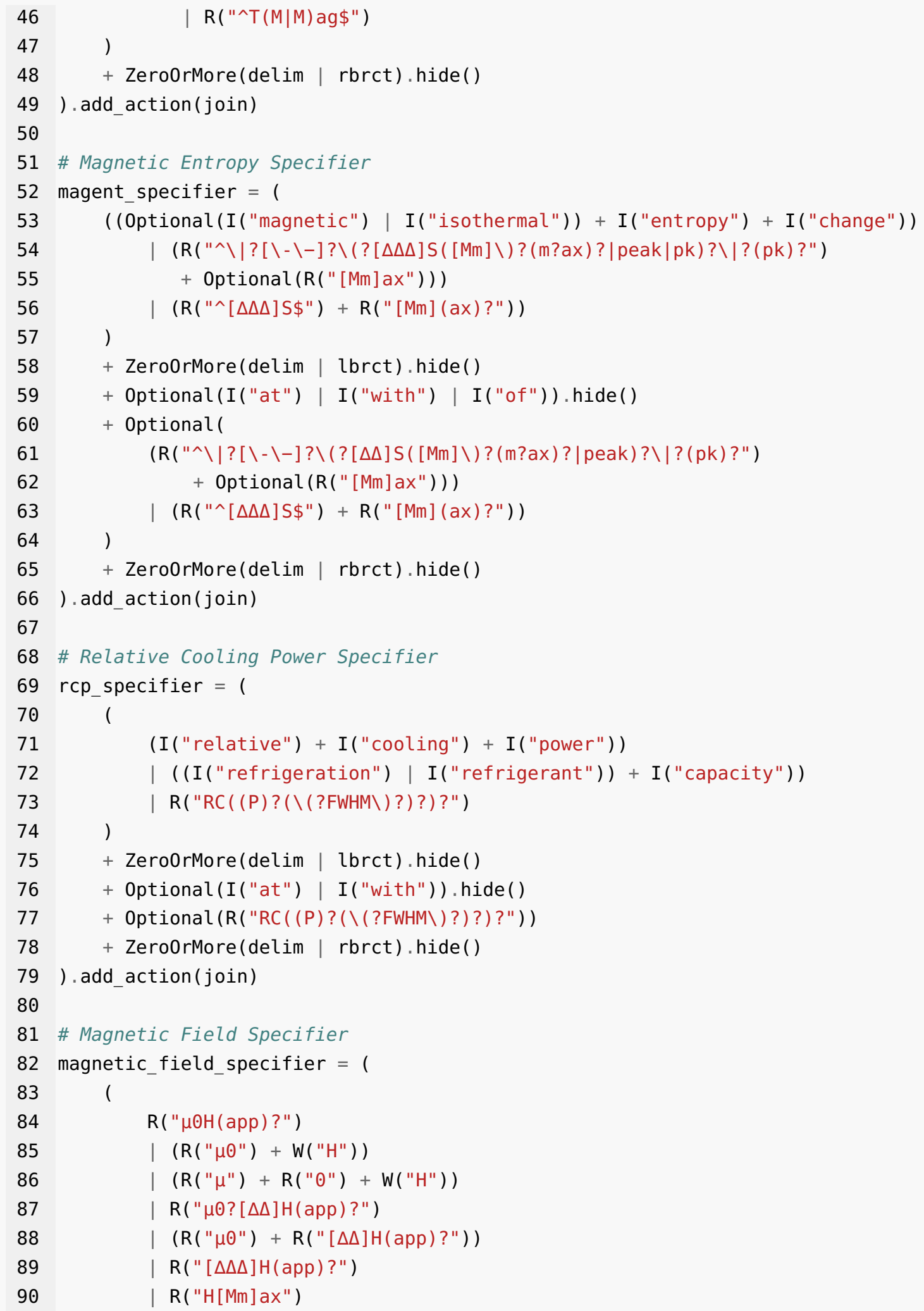




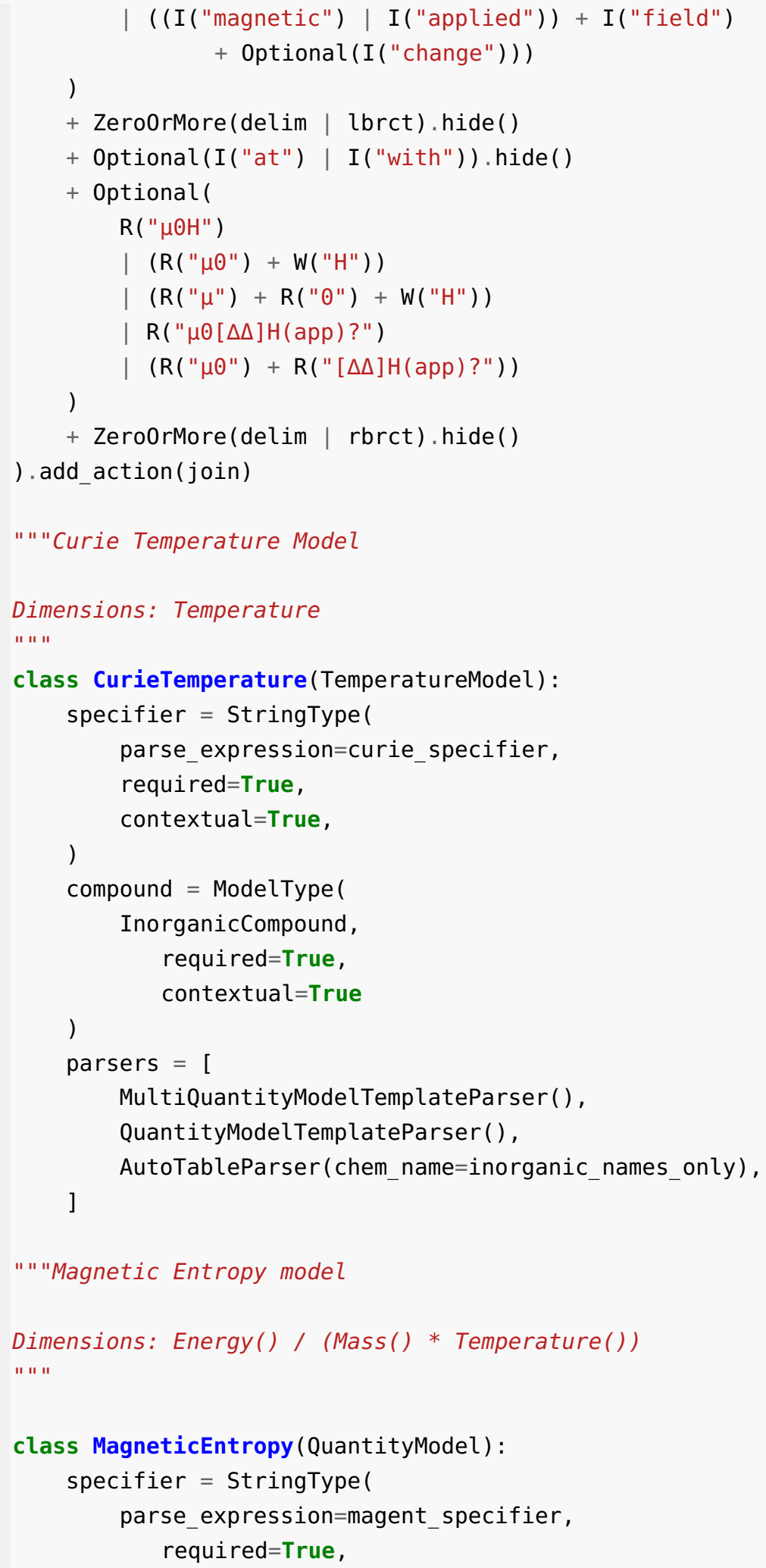




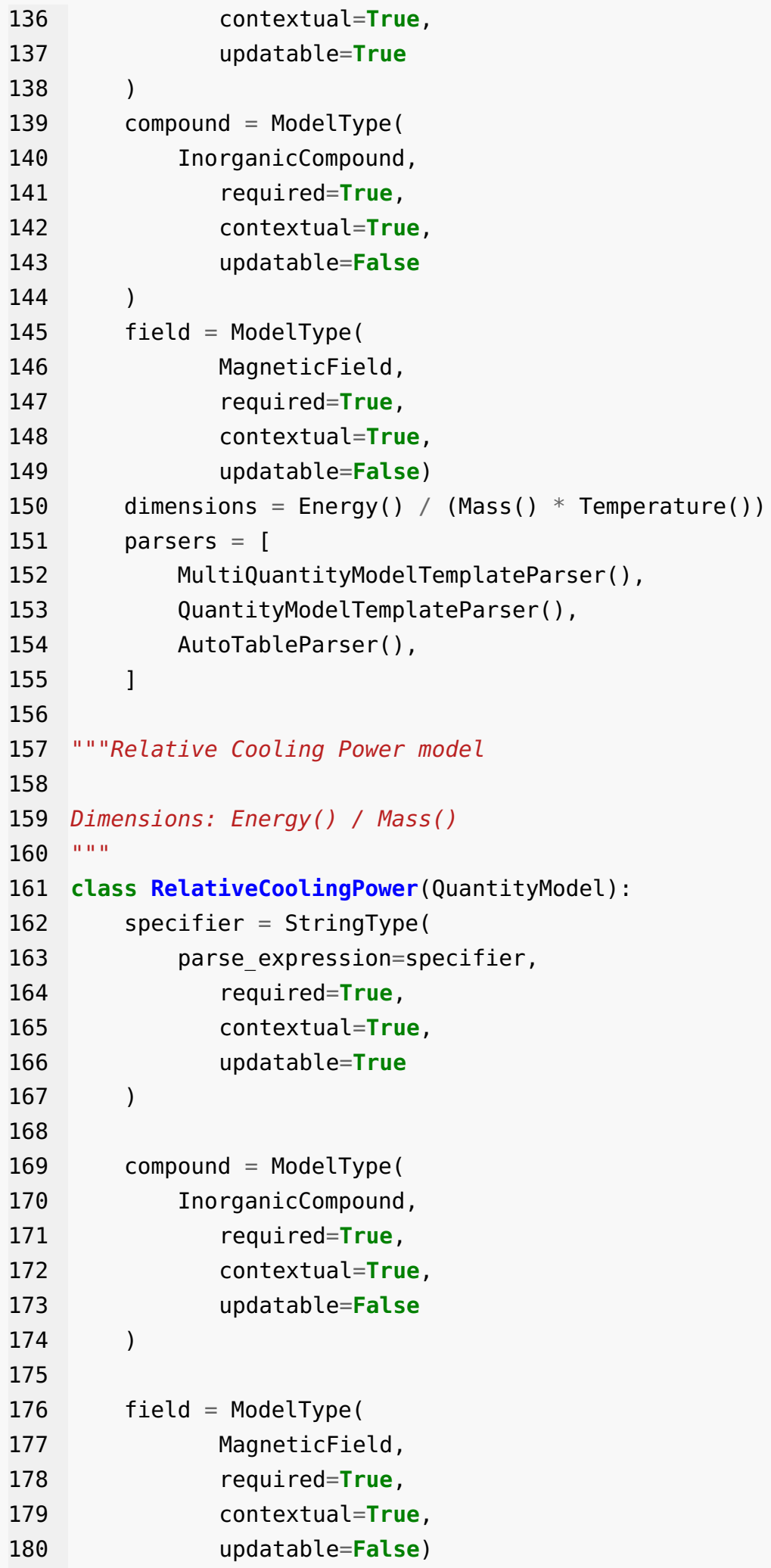


181

182

183

184

185

186

187

188

189

190

" "Magnetic Field Model

191

192

193

194

195

196

197

198

199

200

201

202

203

204

205

206

207

208

Dimensions: MagneticField()

" " " "

class MagneticField(MagneticFieldModel):

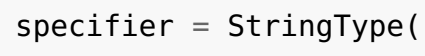

209

] 


\section{Magnetocaloric Regression Model Features}

The table below provides a list of the 58 element-level features and the magnetic field feature used to train the magnetocaloric property prediction models. All element-level features were obtained from the XenonPy python library? .

Each compound is converted to seven composition-weighted feature vectors, using the weighted average, weighted sum, weighted variance, maximum pooling, minimum pooling, geometric mean and harmonic mean. For example, consider a binary compound, $A_{w_{A}} B_{w_{B}}$ with element-level features denoted by $f_{A, i}$ and $f_{B, i}$ for $i=(1, \ldots, 58)$. We then compute the feature descriptors as

$$
\text { Weighted average }=w_{A}^{*} f_{A, i}+w_{B}^{*} f_{B, i}
$$

$$
\text { Weighted sum }=w_{A} f_{A, i}+w_{B} f_{B, i}
$$

$$
\text { Weighted variance }=w_{A}^{*}\left(f_{A, i}-f_{\text {ave }, i}\right)^{2}+w_{B}^{*}\left(f_{B, i}-f_{\text {ave }, i}\right)^{2}
$$

$$
\begin{gathered}
\text { Geometric mean }=\sqrt[w_{A}+w_{B}]{f_{A, i}^{w_{A}} * f_{V, i}^{w_{B}}} \\
\text { Harmonic mean }=\frac{w_{A}+w_{B}}{\frac{1}{f_{A, i}} * w_{A}+\frac{1}{f_{B, i}} * w_{B}}
\end{gathered}
$$

$$
\text { Maximum pooling }=\max _{A, i}, f_{B, i}
$$

$$
\text { Minimum pooling }=\min f_{A, i}, f_{B, i}
$$


Table 1: Element-level feature vectors for the magnetocaloric property prediction models.

\begin{tabular}{|c|c|}
\hline Feature & Description \\
\hline period & Period in the periodic table \\
\hline atomic_number & Number of protons found in the nucleus of an atom \\
\hline mendeleev_number & Atom number in Mendeleevs periodic table \\
\hline atomic_radius & Atomic radius \\
\hline atomic_radius_rahm & Atomic radius by Rahm et al \\
\hline atomic_volume & Atomic volume \\
\hline atomic_weight & The mass of an atom \\
\hline icsd_volume & Atom volume in ICSD database \\
\hline lattice_constant & Physical dimension of unit cells in a crystal lattice \\
\hline vdw_radius & Van der Waals radius \\
\hline vdw_radius_alvarez & Van der Waals radius according to Alvarez \\
\hline vdw_radius_mm3 & Van der Waals radius from the MM3 FF \\
\hline vdw̄e-radius_uff & Van der Waals radius from the UFF \\
\hline covalent_radius_cordero & Covalent radius by Cerdero et al \\
\hline covalent_radius_pyykko & Single bond covalent radius by Pyykko et al \\
\hline covalent_radius_pyykko_double & Double bond covalent radius by Pyykko et al \\
\hline covalent_radius_pyykko_triple & Triple bond covalent radius by Pyykko et al \\
\hline covalent_radius_slater & Covalent radius by Slater \\
\hline c6_gb & C_6 dispersion coefficient in a.u \\
\hline density & Density at $\mathrm{T}=295 \mathrm{~K}$ \\
\hline dipole_polarizability & Dipole polarizability \\
\hline electron_affinity & Electron affinity \\
\hline electron_negativity & Tendency to attract a shared pair of electrons \\
\hline en_allen & Allen's scale of electronegativity \\
\hline en_ghosh & Ghosh's scale of electronegativity \\
\hline en_pauling & Mulliken's scale of electronegativity \\
\hline gs_bandgap & DFT bandgap energy of $\mathrm{T}=0 \mathrm{~K}$ ground state \\
\hline gs_energy & DFT energy per atom of $\mathrm{T}=0 \mathrm{~K}$ ground state \\
\hline gs_est_bcc_latcnt & Estimated BCC lattice parameter \\
\hline gs_est_fcc_latcnt & Estimated FCC lattice parameter \\
\hline gs_mag_moment & DFT magnetic moment of $\mathrm{T}=0 \mathrm{~K}$ ground state \\
\hline gs_volume_per & DFT volume per atom of $\mathrm{T}=0 \mathrm{~K}$ ground state \\
\hline hhi_p & Herfindahl-Hirschman Index production values \\
\hline hhi_r & Herfindahl-Hirschman Index reserves values \\
\hline specific_heat & Specific heat at $20^{\circ} \mathrm{C}$ \\
\hline first_ion_en & First ionisation energy \\
\hline fusion_enthalpy & Fusion heat \\
\hline heat_of_formation & Heat of formation \\
\hline heat_capacity_mass & Mass specific heat capacity \\
\hline heat_capacity_molar & Molar specific heat capacity \\
\hline boiling_point & Boiling temperature \\
\hline bulk_modulus & Bulk modulus \\
\hline melting_point & Melting point \\
\hline thermal_conductivity & Thermal conductivity at $25^{\circ} \mathrm{C}$ \\
\hline sound_velocity & Speed of sound \\
\hline Polarizability & Ability to form instantaneous dipoles \\
\hline poissons_ratio & Poissons ratio \\
\hline molar_volume & Molar volume \\
\hline num_unfilled & Total unfilled electrons \\
\hline num_valence & Total valence electrons \\
\hline num_d_unfilled & Unfilled electrons in d shell \\
\hline num_d_valence & Valence electrons in d shell \\
\hline num_f_unfilled & Unfilled electrons in $\mathrm{f}$ shell \\
\hline num_f_valence & Valence electrons in $\mathrm{f}$ shell \\
\hline num_p_unfilled & Unfilled electrons in $\mathrm{p}$ shell \\
\hline
\end{tabular}


Feature

num_p_valence

num_s_unfilled

num_s_valence applied_field
Description

Valence electrons in $\mathrm{p}$ shell

Unfilled electrons in s shell

Valence electrons in s shell

Field measurement for RCP and entropy models 